\title{
La reparación y el cuidado de sí mismo desde la psicoterapia psicodramática bipersonal
}

\section{Self-repair and self-care based on the bipersonal psychodramatic psychotherapy}

\author{
Silvia Monzón Reviejo \\ Centro QUIPÚ Psicoterapia y Salud Mental, España
}

\begin{abstract}
Resumen: El trabajo que expongo tiene el objetivo de mostrar cómo realizo el trabajo de reparación de las heridas sufridas en la biografía de los pacientes desde la psicoterapia psicodramática bipersonal. Partiendo de la complementariedad del modelo psicodinámico y psicodramático, y sin desvirtuar ambas escuelas, trato de hacer posible la aplicación de diferentes modalidades de intervención para el trabajo de reparación y la construcción del cuidado de sí mismo en los pacientes.

La finalidad es poder realizar un manejo adecuado de la conflictiva del paciente sabiendo cuándo y cómo pueden empezar a identificar, elaborar y reparar las heridas opresivas y carenciales creadas y agravadas a lo largo de su biografía.

Palabras clave: Psicoterapia, psicodrama bipersonal, reparación psíquica, heridas evolutivas.

Abstract: This paper is intended to show how to repair the wounds suffered by patients during their lives using bipersonal psychodramatic psychotherapy. Based on the psychodynamic and psychodramatic complementary model, without undermining both schools, I try to make it possible to implement different intervention methods to allow patients to for repairing and constructing care of themselves. The purpose is to be able to carry out an adequate management of the patient's conflict, knowing when and how they can start to identify, elaborate, and repair the oppressive and deficiency wounds created and aggravated during their lives.

Keywords: Psychotherapy, bipersonal psychodrama, psychic repair.
\end{abstract}

Silvia Monzón Reviejo es Doctora en Psicología. Psicóloga Especialista en Psicoterapia. (EFPA). Miembro Titular del Centro QUIPÚ Psicoterapia y Salud Mental. Secretaria y coordinadora de Revista Intersubjetivo.

La correspondencia sobre este artículo debe enviarse a la primera autora al E-mail: info@silviamonzon.com

(cc) BY-NC-ND Este es un artículo Open Access bajo la licencia CC BY-NC-ND. 
A la hora de cerrar heridas desde la psicoterapia, lo primero que queremos es conocer cuáles son las heridas a reparar, de qué naturaleza son. En segundo lugar, cuándo es el momento de comenzar el proceso de reparación de esas heridas. Y, en tercer lugar, pensar en cómo intervenimos para que se produzca la reconstrucción.

Cerrar las heridas que se han ido agravando a lo largo de la historia del paciente, en psicoterapia se materializa a través de la elaboración y restauración. Reparar las ambivalencias, las heridas sufridas, los vínculos dolorosos y conflictivos lleva tiempo. Muchas veces, desde la angustia del paciente pero también del terapeuta, queremos empezar a reparar desde el comienzo del tratamiento, sabiendo que la reparación solo se puede alcanzar en periodos medios y avanzados del mismo.

Generar cambios se consigue con relativa facilidad, pero reparar vivencias dolorosas, confusas y/o ambivalentes y crear estabilidad interna lleva su tiempo y precisa de su momento. No solo necesitamos la construcción del vínculo con el paciente y respetar el periodo de asimilación de lo sucedido para poder llegar a la reparación, además nos encontramos con las resistencias que impiden al paciente recibir cosas buenas de los demás y de sí mismo, y que les imposibilitan reparar lo vivido.

Las personas, cuando acuden a psicoterapia, buscan ayuda y quieren conscientemente reconstruir su vida, quitarse el sufrimiento y resolver los problemas, pero seguidamente podemos observar cómo se activa en ellos una serie de mecanismos que se lo impiden. Cuando el paciente se enfrenta a la situación psicoterapéutica, precisamente porque no quiere sufrir, no quiere sentir más dolor, esta hace peligrar su equilibrio defensivo y se produce automáticamente y de manera inconsciente un reforzamiento de sus miedos y defensas. Son resistencias a descubrir y sentir vivencias que le puedan doler, pero también son resistencias a cambiar cierto grado de seguridad que, a pesar de su sufrimiento, le permite al paciente encontrarse en un equilibrio, aunque lo haya alcanzado a costa de renuncias y limitaciones (Monzón, 2012).

Concluyendo, la reparación y el cuidado de sí mismo se lleva a cabo cuando son vencidos y elaborados los miedos y las resistencias en el curso del tratamiento. Si estas defensas no se levantan, en psicoterapia no hay posibilidad de cambio, porque no podemos acceder a las distintas fases del proceso psicoterapéutico que inciden en el cambio y que se resumen en construir en el paciente la disposición afectiva a mirar hacia atrás, en vivenciar y confiar en nosotros, y por último, en poder elaborar lo sucedido y empezar a reparar.

\section{Qué y cómo reparar}

Partiendo de la concepción psicodinámica de conflicto y déficit psíquico, donde el síntoma que presenta el paciente representa la expresión de un conflicto y un déficit sin resolver y que ha sido gestado en una serie de etapas que ha vivido conflictivamente, la restauración va a tratar de reparar cada una de estas vivencias conflictivas padecidas por el paciente.

Siguiendo las ideas expuestas por Villamarzo (1987), sobre todo en los pacientes melancólicos, paranoides y obsesivos, la reparación consiste en la exposición a las dificultades para entregarse a la situación de disfrute de la vivencia restauradora y dejarse llevar por la satisfacción del tratamiento. Citando algunas fantasías temidas de los pacientes y/o algunos miedos a repetir las vivencias sufridas anteriormente, los temores que crean estas resistencias a elaborar y restaurar están relacionados con no sentirse con derechos a entregarse al disfrute, a reparar ni a cuidarse a sí mismos.

En el caso de los pacientes melancólicos, también puede ser por el miedo a que se vuelva a repetir un rechazo si se dejan abandonar por la situación de goce con el terapeuta. Si se trata de pacientes con personalidad obsesiva, no se permiten la restauración por fuertes sentimientos de culpa.

En las organizaciones limítrofes les cuesta internalizar, cuidarse o sentirse con derecho a recibir o a disfrutar, entre otras cuestiones, por sentirse malas personas: «Soy demasiado mala persona como para darme derecho a estar bien». En el caso del paranoide, se resiste a acercarse y recibir del terapeuta, ya que a niveles más profundos lo vive como perseguidor. Cuando se trata de resistencias narcisistas, la entrega a la restauración la viven con humillación, ya que sería como admitir que no lo tienen todo y que el otro tiene algo que él no tiene, comenzando a rivalizar, a agredir el tratamiento o a desvalorizarlo, puesto que lo viven como una derrota.

En la tabla 1 podemos ver la clasificación de las heridas evolutivas a reparar propuesta por Villamarzo (1987). 


\section{Tabla 1. Heridas Evolutivas}

POR DEFECTO (carencia, déficit):

Seguridad -esquizoides-

Afecto -melancólicos-

Valoración -narcisistas-
POR EXCESO (opresión, culpa):

Exigencias -obsesivos-

Sobreprotección -fóbicos-

Culpabilización -histeria-

Fuente: (Villamarzo, 1987).

De esta manera, conociendo las heridas a reparar, el siguiente paso para el trabajo de la restauración del sí mismo desde la psicoterapia psicodramática bipersonal, sería conocer qué tipos de intervenciones verbales y en escena psicodramática se aplicarían. Desde el psicodrama bipersonal, término creado por Dalmiro Manuel Bustos, se designa como psicoterapia psicodramática bipersonal al abordaje terapéutico que tiene su origen en el psicodrama, pero que no se sirve de yoes-auxiliares y atiende a un solo paciente cada vez, creando así una relación bipersonal, o sea, un paciente y un terapeuta (Cuckier, 1992). Este modo de hacer psicoterapia corresponde a una modalidad dentro del psicodrama, en coherencia con muchos postulados de Moreno, el fundador del psicodrama, pero donde lo que se retiene de los fenómenos observables lo comprendemos gracias al pensamiento psicoanalítico (Monzón, 2011).

En el trabajo de la reparación, desde el psicodrama bipersonal, las intervenciones verbales de tipo psicodinámico son intervenciones de apoyo, es decir, en primer lugar señalamos la dificultad para decirse cosas buenas a sí mismo, la dificultad de darse derechos o de distanciarse de las figuras negativas de la infancia. Y en segundo lugar, una vez vencida la resistencia a hacerlo, le invitamos al paciente a decirse cosas buenas, a darse derechos, a distanciarse de las figuras parentales.

Cuando realizamos técnicas psicodramáticas en psicoterapia psicodramática bipersonal para facilitar la reparación desde una escena vivenciada por el paciente en el espacio psicodramático, nos trasladamos del espacio verbal (común a cualquier psicoterapia relacional, sentados paciente y terapeuta) y nos levantamos para dirigirnos hacia el espacio psicodramático (espacio utilizado para aplicar las técnicas de psicodrama). Para ello, necesitamos dos sillas y varios almohadones. Al paciente se le coloca en el espacio psicodramático en una silla, y enfrente, en otra silla, colocamos un cojín (objeto simbólico representante de la conducta, conflicto o emoción a reparar).

Durante las dramatizaciones que realiza el paciente, el terapeuta no efectúa ningún cambio de rol, permanece junto al paciente desde su función de terapeuta, a su lado; ni tampoco es el que le dice cosas buenas o quien le da derechos al paciente. No emitimos directamente un juicio de valor con respecto a que sean buenos o malos sus temores o sus fantasías. De esta forma, señalando los temores, las dificultades e invitando a actuarlas o decirlas, los pacientes introyectan nuestra intervención como «luego tengo cosas buenas», intentando vencer las dificultades y trabajar con sus resistencias.

\section{La reparación desde la psicoterapia psicodramática bipersonal}

Desde la psicoterapia psicodramática bipersonal, el trabajo de las resistencias a la elaboración y restauración, también denominado catarsis de integración con fines a la elaboración (Bustos, 1985), se lleva a cabo por las vías dramática y verbal. Ambas, nos aportan perspectivas diferentes.

Como comentaba anteriormente, con las intervenciones psicodinámicas verbales, el objetivo psicoterapéutico para este tipo de resistencias a la restauración es utilizar las intervenciones de apoyo para ir solventando las resistencias a la restauración, vamos señalando verbalmente las dificultades que tenga el paciente según sea la naturaleza de las resistencias. 
Estas intervenciones verbales se pueden realizar tras una escena a reparar que ya ha verbalizado el paciente o antes de acceder al espacio psicodramático para facilitar la escena a trabajar en concreto.

Por ejemplo, si las resistencias son frente a la dificultad para darse derechos a sí mismo y de forma verbal vemos que aumenta la resistencia o que no lo está recogiendo emocionalmente, podemos utilizar en el espacio psicodramático la técnica del espejo o la del observador externo. Estas técnicas consisten en colocar frente a la persona, en el espacio psicodramático, un objeto simbólico que les represente a sí mismos y pedirles que se observen desde fuera, como si fuesen observadores externos, o que se observen a ellos mismos como si estuviesen mirándose en el espejo, para seguidamente preguntarles cuáles considerarían que son las dificultades que tienen para decirse cosas buenas, o las resistencias para cuidarse a sí mismos, ser buena madre consigo mismos o para darse derechos. Según sea la naturaleza de las resistencias, señalamos «la dificultad de decirse cosas buenas a sí mismo», «la dificultad de darse derechos», «de distanciarse de las figuras negativas de la infancia», «dificultades para darse seguridad, afecto o/y valoración»o las «dificultades para quitarse exigencias, exceso de protección o para retirarse la culpa» (Monzón, 2011).

La técnica del espejo es útil en la medida que el paciente puede verse mejor y se distancia un poco de la angustia, pudiendo empezar a entender lo que le ocurre y poder manejar mejor la escena. Utilizando la visualización y el soliloquio y acompañado por la entrevista del terapeuta, el paciente tiene la viveza de la escena y la distancia de la observación para conocer y elaborar mejor las dificultades para poder cuidarse o reparar las escenas y las heridas producidas en su biografía.

La técnica del espejo o la del observador externo, la visualización y el soliloquio, son técnicas utilizadas en el espacio psicodramático para identificar la dificultad o la resistencia para, en este caso, la reparación y el cuidado de sí mismo. Cuando queremos dar paso no solo a identificar y elaborar sino además a profundizar en esa reconstrucción de uno mismo, la técnica psicodramática más adecuada es la denominada técnica de $E l$ yo adulto y el yo niño, que consiste en elaborar y reparar las vivencias dolorosas de los pacientes.

A lo largo de estos años como psicoterapeuta psicodramática he podido observar que esta técnica facilita un espacio operativo donde el paciente puede construir una imagen de cuidado de sí mismo, una figura interna afectiva, valiosa y protectora que se activa en aquellas situaciones angustiantes y que cuida del niño o de la niña asustada que aparece ante ellas.

\section{Construyendo el cuidado de sí mismo a través de la técnica el yo adulto y el yo niño}

La técnica psicodramática conceptualizada como el yo adulto y el yo niño ha sido utilizada de diversas formas y en etapas distintas en el proceso psicodramático. La psicodramatista americana Marcia Karp, en 1990, fue la creadora de esta técnica, que utilizó como un juego de caldeamiento. Rosa Cuckier (1992) conoce esta técnica a partir de ella y la desarrolla por medio del psicodrama interno, con el objetivo de sensibilizar al paciente en relación a sus necesidades infantiles.

Denominada también como el yo grande y el yo pequeño, esta técnica se encuentra integrada como parte final de los juegos que desean elaborar la matriz de las conductas defensivas (Bustos, 1985). Entendemos como conductas defensivas una serie de funciones reactivas que el paciente desarrolla a lo largo de su vida con la finalidad de controlar su angustia. Estas defensas se forjan habitualmente en la infancia, donde los niños las emplean para protegerse de los conflictos y déficits que se hayan producido a través del vínculo con el adulto.

Además de tener efectos catárticos, y de ser utilizada como caldeamiento e identificación de la matriz de las heridas opresivas y/o carenciales que se han ido agravando a lo largo de la historia del paciente, también es una técnica aplicada con fines elaborativos y reconstructivos, cuyo objetivo es cerrar estas heridas, de manera que permita elaborar y reparar los conflictos y las carencias producidas en la biografía del paciente.

Antes de establecer cuándo y cómo utilizarla, quisiera matizar que cuando utilizamos dicha técnica no se aplica de manera aislada, sino que forma parte del proceso psicoterapéutico en el que se encuentra el paciente. En función de este y de la patología que representa el sujeto, podremos saber cómo y en qué momento emplearla.

Partiendo de esta base, la técnica psicodramática el yo adulto y el yo niño se puede aplicar en las siguientes 
situaciones: a) Sensibilizar al paciente con sus necesidades infantiles, b) Trabajo de la conducta sintomática y la motivación que lo genera y c) Elaboración de una escena no resuelta que nos muestra el paciente en sesión.

\section{Sensibilizar al paciente con sus necesidades infantiles}

Se trata de recuperar, a través del psicodrama interno, momentos del pasado del paciente y de enfrentándolos con el presente, con el objetivo de sensibilizarle en relación con sus necesidades infantiles (Cuckier, 1992).

Este primer caso, como decía en líneas anteriores, es una opción más directiva y de caldeamiento para un trabajo elaborativo posterior. Es una medida que no suelo utilizar habitualmente en mi trabajo con psicodrama bipersonal, por lo que no es el objetivo de este artículo. El trabajo psicoterapéutico que realizo y expongo en este trabajo corresponde habitualmente a las opciones segunda y tercera, que a continuación expongo, es decir, como elaboración y reconstrucción de las heridas opresivas y carenciales producidas en el paciente.

\section{Trabajo de la conducta sintomática y la motivación que lo genera}

La técnica de el yo adulto y el yo niño la aplico a partir de una conducta defensiva y partiendo de la técnica de la concretización -explicada más adelante-, o bien una vez que han aparecido aquellas situaciones originarias de una carencia y/o conflicto que el paciente ha sufrido, ya sean estas verbalizadas por el paciente o visualizadas en el espacio psicodramático.

Cuando partimos de pedirle al paciente que visualice una situación en concreto, lo hace desde su rol adulto, visualizando la escena de dos formas diferentes: ya sea con los ojos cerrados (psicodrama interno) o bien visualizando la escena frente a él a través de un objeto simbólico que la represente (visualiza la escena a través de un cojín o de la pared, como si tuviera enfrente una pantalla de cine).

De esta forma, llevamos a cabo el trabajo de la escena a partir de la creación de dos roles: el rol de adulto y el rol de niño. En un primer momento, el paciente ejerce el rol de adulto, y desde ese rol adulto visualiza la escena que está enfrente de él, en la que se desarrolla el rol de niño.

El objetivo es que realice un encuentro entre su ser adulto y ese niño atrapado en aquella situación no resuelta para, en último lugar, ofrecer a ese niño, desde su rol adulto, lo que hubiera necesitado recibir en aquel momento. Una vez expresado lo que le hubiera gustado dar o decir a ese niño o lo que cree que hubiera necesitado ese niño en aquella situación, se cambia de rol (y físicamente también se cambia de posición y de silla) y se coloca en el rol de niño, permitiéndose experimentar la vivencia de esos mensajes enviados por el rol de adulto.

El yo adulto es aquel rol que se crea para construir una representación mental interna de cuidado; una figura afectiva, valiosa y protectora que cuide del niño asustado. El colocarse y ponerse en la piel del yo niño permite llevarnos al lugar donde las necesidades afectivas y las carencias se organizaron, al lugar constituyente y a la matriz constitutiva del sufrimiento, de la psico(pato)logía (Herranz, 2012).

Cuando utilizo la técnica partiendo del síntoma que trae el paciente, primero aplico en el espacio psicodramático la técnica de la concretización del síntoma en un personaje. Para ello, necesitamos dos sillas y varios almohadones. Al paciente se le coloca en el espacio psicodramático en una silla, y enfrente, en otra silla, colocamos un cojín (objeto simbólico representante de la conducta sintomática).

A continuación, transformamos el síntoma en un personaje simbólico que lo va a representar. Queremos que el paciente se imagine cómo es ese síntoma, qué forma y tamaño tiene esa conducta sintomática que visualiza frente a él y qué porcentaje ocupa en su vida.

El siguiente paso consiste en cambiar de rol al paciente (física y mentalmente) y colocarlo en el rol del síntoma. Ocupando el paciente el rol de síntoma, el terapeuta, que permanece de pie y a su lado, le pregunta acerca del tiempo, la edad y el motivo por el que aparece este síntoma en su vida. El objetivo es encontrar el nacimiento del síntoma, de la defensa, cuál era la necesidad del paciente en ese momento y cuál es el conflicto que lo originó.

La técnica de la concretización termina cuando recorremos la escena infantil (psicodrama vertical) que ha traído el paciente. Visualizando frente a él qué ocurría en el momento en que aparece el síntoma (personaje), 
a través de la técnica de el yo grande y el yo pequeño damos paso a la reconstrucción de la escena infantil. Seguidamente, expongo el trabajo realizado a través de la descripción de varias viñetas clínicas.

Ejemplo clínico 1. El yo adulto reparando el abuso sexual paternofilial. En primer lugar, quiero mostrar una viñeta clínica con el trabajo psicoterapéutico realizado en un caso de abuso sexual de un padre hacia su hija. En este caso, se trata de una paciente adulta con importantes heridas melancólicas. A esta paciente, sin sentirse con derecho a desear tras haber vivido abusos sexuales en su infancia, en el momento psicoterapéutico en el que voy a proceder a la reparación de sus vivencias, la coloco en el espacio psicodramático como observadora externa de la escena infantil del abuso sexual verbalizado.

El trabajo, en un primer momento, consiste en narrar y vivenciar la escena infantil como si fuera una observadora externa, que visualice frente a ella qué ocurría en ese instante. A continuación, la pregunto, mirando y observando la paciente desde fuera la escena, cómo se sentía esa niña, con quién estaba, qué le decían o qué no le decían y qué hizo.

La técnica del observador externo es fundamental realizarla en este tipo de vivencias traumáticas de abuso, pero para poder repararla hay que ir despacio, colocando primero a la paciente fuera de la escena y que no se inunde emocionalmente. Para elaborar y reparar la escena se necesita un mínimo distanciamiento y contención afectiva.

Una vez que tenemos la escena visualizada, el siguiente paso es la reparación de la escena con la técnica de el yo adulto y el yo niño.

En este mismo caso, a la mitad aproximadamente del proceso terapéutico, a través del vínculo y las técnicas utilizadas, ya puede comenzar a verbalizar las escenas de abuso, siendo la angustia el contenido fundamental a tratar. Colocamos la angustia en un cojín que la simbolice. Me dice que la cara o la persona que le viene a la cabeza es su padre; y la escena, cuando ella es pequeña.

La propongo que describa la situación desde fuera, como si fuese una película de su vida. Ella comenta que en la escena están ella y su padre, y lo que le pide su padre (conductas de masturbación), mientras le dice que ella es especial para él, que es la preferida y que no tiene que decir nada.

La pido que vea desde fuera esa situación, desde la mujer adulta que está conmigo, y que, como si tuviera a esa niña enfrente de ella, se ocupe de ella. Se le pregunta qué quiere hacer con esa niña, qué la sale hacer en ese momento, y me dice que sacarla de la escena. Despacio, la saca de la escena y la lleva con sus hermanos a otra habitación a jugar. Juega allí con ellos. La sugiero que piense si quiere decirle algo a esa niña en relación a lo que ha pasado y ahora que está más segura, que vea qué puede necesitar de ella la niña entonces.

El mensaje que le transmite la paciente, desde su rol adulto a su rol de niña abusada, es el siguiente: «Tú no tienes la culpa, no sabes qué pasa, ahora ya estás a salvo, con tus hermanos, a jugar». La invito a que le explique a la niña por qué ella no tiene la culpa. La paciente le dice que no tiene la culpa de lo que está pasando, ella no entiende qué pasa.

A continuación, la digo que se coloque en el lugar de la niña y que vea cómo se siente con lo que ha pasado. Me dice que confusa, que no sabe qué pasa, que piensa que ella le está provocando a él: "Confusa porque, por un lado, siento asco, pero por otro me siento bien por ser especial, la preferida, de estar con él y de provocarle, como decía mi padre».

Objetivos y reflexiones terapéuticas: « ¿Pero qué es lo que entiende esta niña? ¿Entiende el significado del acto sexual? ¿Qué entiende esta niña de actos y conductas sexuales? Nada. ¿Ser la preferida implica el acto sexual? Una cosa es desear ocupar el lugar de ser la preferida, otra distinta el acto sexual». Por tanto, el objetivo es que la paciente comience a entender que una niña necesita a su padre, que una niña también tiene sexualidad, pero que eso no quiere decir que esté provocando ni que esa niña sea horrible.

El siguiente paso consistía en preguntar a la paciente si su padre tenía derecho a hacer lo que hizo con ella, y en ayudarla a colocarse en su rol de adulta, enseñándola a diferenciar la necesidad de ser la preferida, de someterse o de tener que ser abusada: «Para ser la preferida, para ser la elegida, ¿hace falta hacer esto?»

Desde su rol de cuidado, de adulta, la paciente se dirige a la niña y verbaliza lo siguiente: «No tienes la culpa, en realidad tú no le provocabas, eras objeto de manipulación, te hacía creer eso para quitarse y conseguir lo que quería, tu padre no te quiere». Dirigiéndose simbólicamente hacia la figura del padre, le espeta: «Nunca 
me has querido, no has sido un buen padre, me das asco, me has jodido la vida». Luego, desde su rol de niña, verbaliza: «No quiero hacer esto, no me gusta, me quiero ir».

Por último, le pregunto si va a seguir permitiendo que le perjudique en su vida. La paciente afirma: «No, no voy a permitirlo, no voy a permitirle que me joda la vida».

Ejemplo clínico 2. El yo adulto reparando la culpa en las vivencias de abuso sexual entre hermanos (abuso incestuoso). Una paciente adolescente se había colocado en un lugar donde se sentía culpable por desear y por excitarse. Siguiendo los pasos anteriormente comentados a través de la técnica de concretización, en el espacio psicodramático damos forma a esa culpa. La culpa viene desde hace muchos años, siendo ella pequeña.

Narra y vivencia la escena infantil como si fuera un observador externo y visualiza frente a ella qué ocurría en ese momento. En seguida, vemos cuándo apareció la culpa en su vida, cómo se sentía, con quién estaba, qué le decían o qué no le decían y qué hizo.

Habla, en primer lugar, de una imagen con su hermana donde había frotamientos con la ropa puesta. Más adelante, comenta que ella empezó a hacerlo también con su hermano pequeño. Sin embargo, con su hermano (tres años menor) comenta escenas donde, en uno de esos frotamientos, van más allá del juego de exploración evolutiva, realizando un intento de sexo oral con él. En ese momento, la paciente siendo niña se asusta, se para y le dice a su hermano: «Bueno, esto nunca ha pasado, vete a por un vaso de agua».

Una vez narrada y visualizada la escena como observadora externa de la situación, intervengo en la misma y la pregunto qué quiere hacer con la niña en aquel momento que está describiendo y vivenciando. Me dice que no quiere quitarla la culpa porque lo que hizo estaba muy mal. La pregunto qué edad tenía y si sabía lo que hacía como niña. Me dice que prefiere no saber porque, además, también había penetraciones: «Todo esto lo hacíamos los tres, con mis primos... y muchas veces. Me siento culpable con todo».

A medida que se afianza la relación terapéutica, el paciente va abriendo paso a aquellas vivencias más antiguas sin resolver. Cuando rescatamos estas situaciones en escena para repararlas, siempre nos preguntamos qué es lo que el paciente necesita para poder cerrar esta vivencia angustiosa y no volverla a repetir en sus relaciones y/o a través de sus síntomas.

En este caso, partimos del descuido parental, que hace que el vínculo entre hermanos se intensifique. La ausencia de los padres puede generar que los límites entre los hermanos puedan perderse, y que los niños que han perdido el cuidado y los límites de los padres se refugien en lo sexual.

Objetivos terapéuticos: ¿Dónde estaban sus padres? La culpa viene porque ha hecho daño al hermano, porque todos participaban y por el placer ligado a la culpa que experimenta, que es naturalmente intolerable.

La intervención desde el psicodrama bipersonal parte del cambio de roles simbólicos con los padres: ¿Qué pensarían sus padres sobre esta situación entre hermanos? Si la culpan: «¿Y ustedes qué la enseñaron?»El objetivo es tomar conciencia de esa parte de desatención: «Te dejaron descubrir la sexualidad sin guía. Es una sexualidad descubierta hasta ahora como si fueras una niña, sin guía».

En el caso de que no le culpen los padres, el temor ya es más interno y está ligado a las fantasías de «todo es posible». Dime: «Qué es posible ahora para ti, qué es posible para esta niña, pero sin insultarla».

Haciendo el ejercicio psicodramático, comenta que sus padres no estaban pero que, si hubieran estado, «mi padre se hubiera ido y le hubiera dejado el marrón a mi madre, que me hubiera regañado, pero luego como si nada... no lo sé... mi madre no estaría... no lo sé».

La siguiente intervención va encaminada a redirigir la escena con el hermano, creando en la paciente un rol de adulto: «¿Qué hubieras hecho tú si fueras su padre o su madre? ¿Qué necesitaba esta niña?» «Desde tu rol de adulta, mira a ver si puedes llevar a la niña hacia otra actividad, sin regañar e informando de lo que se debe hacer o no. Así ya puede empezar a saber la niña que eso es inapropiado».

En este caso, en vez de seguir culpando a esa niña y repetir el patrón, creamos un yo adulto y construimos lo que necesitaba esa niña entonces (la presencia de unos padres, y no una voz que habla desde el castigo). A través de preguntas, se le invita a reflexionar: «¿Qué hubieras hecho si fueras padre o madre?»

Así, la vamos guiando en ese proceso, donde en vez de regañar o no hacer nada como padres, la paciente es la que debe ir informando a la niña, a través de la escena y enfrente de ella, de lo que se puede hacer o no, 
redirigiendo su actividad a otra cosa para que empiece a ver lo que es apropiado e inapropiado y, por último, preguntándola dónde lo aprendió.

Ejemplo clínico 3. Cuando no existe el yo adulto: creando una capacidad mínima de cuidado y protección. Cuando las heridas han sido muy carenciales y han existido importantes déficits en casi todas las áreas (afectiva, de valoración y seguridad), muchas veces no tienen una representación de cuidado de sí mismos y no saben ni pueden colocarse en el rol de adulto.

En el caso de una paciente adulta con patología borderline que no había recibido apenas mensajes de cuidado por parte de su familia y que sentía mucha rabia hacia ellos, ante la incapacidad de originar mensajes de cuidado desde su rol adulto, que apenas lo tiene construido, se le pide, sobre todo al principio, que busque a aquellas personas que lo pudieran encontrar por ella. En muchas situaciones en las que se sentía sola en su familia, se intentaba acercar a la gente y buscar mensajes de cuidado.

Partiendo de la técnica de la concretización en un personaje, en esta situación, el personaje sintomático es la agresividad, y es una defensa ante el sentimiento de rechazo: «Es un gremlin, que viene para que, si se enfada, por lo menos ya tenga una excusa para entender que los demás no quieran estar con ella». Muchas veces es mejor pensar que los demás no quieren estar con uno mismo porque es agresivo, que pensar que se le rechaza por lo que uno es.

En aquellas vivencias de carencias afectivas, de seguridad y/o de valoración, con el objetivo de crear una imagen interna de cuidado de sí mismos y reparar las carencias producidas, a través de las escenas realizamos los siguientes pasos, que a continuación expongo (Monzón, 2007):

1. Que busquen figuras que les protejan, que les cuiden.

2. Que busquen aquellos mensajes de tranquilidad y protección que escucharían de esas personas.

3. Si no tienen esa figura, no pueden o no saben hacerlo, que busquen a alguien que lo pudiera hacer por ellos.

4. Si no hay ninguna figura, que se lo inventen o que busquen un objeto mágico.

La búsqueda de una figura inventada o un objeto mágico -objeto transicional- permite, por el momento, que empiecen a buscar una representación simbólica de cuidado y protección, primero desde fuera, a través de los demás, para después poder cuidarse a sí mismo e internalizar esa figura de protección como propia.

Partiendo desde otro lugar, de otra manera de pedir a los demás, se le invita a la paciente a ver qué le gustaría pedir a los otros, de un modo que no sea agrediendo, qué es lo que necesita de ellos en este momento:

P: «Cariño, pero es que nadie me lo da...»

T: «Nadie te lo da como tú quieres... ¿ io nadie te lo da?»

P: «Como yo quiero... no sé... ay, no sé... sí... esta y la otra me lo dan... pero es que...»

T: «Mira a ver de quién crees que podrías recibir lo que necesitas en este momento.»

P: «De nadie... de nadie... nadie me lo puede dar...»

T: «Pues, si te parece, nos lo vamos a inventar. Dime, ¿cómo te imaginas a esa persona que te da lo que tú pides?»

P: «Pues vieja, sabia, como la abuela de Piolín.»

T: «¿Qué te diría?»

$\mathrm{P}$ : «Que no me preocupe, que ya se pasará, que me voy a poner bien... y que me quede sola, que no dé tanto a los demás.»

P: «Ya lo que me faltaba, tener que inventarme amigos...»

T: «No es inventarte amigos, es permitirte una fantasía de aceptación, de sentirte cuidada.»

En periodos medios y avanzados de tratamiento, una vez construido el sentimiento de presencia y de existencia del otro, los pacientes ya tienen una mínima contención de sí mismos, de cuidado, y ya podemos empezar a trabajar para que sea la persona, desde su parte adulta, la que comience a cuidarse.

Comienzan a aparecer muchas situaciones de reparación de las necesidades afectivas. La paciente, siendo una niña y habiendo relatado una escena donde expresaba que necesitaba más cariño y comprensión de lo que recibía, expresaba que sus padres jamás harían muchas cosas por ella, que no apostarían por ella, que sabe que la quieren menos y que nunca la hicieron caso: «Mi padre ya me compara con el peor de mis enemigos, y me 
lo decía mientras se fumaba un puro. No se han preocupado por mí nunca». En este punto, en psicodrama bipersonal se la coloca un cojín que la representa a ella, y se le dice que mire a ver si quiere, aquí y ahora, desde su parte adulta, darle a esa niña lo que no la han dado.

\section{Elaboración de escena no resuelta que nos muestra el paciente en sesión}

Por último, damos paso a la tercera opción del trabajo de reparación del yo adulto: cuando la técnica se utiliza en el espacio psicodramático tras una situación sin resolver, que el paciente previamente verbaliza en sesión con el objetivo de repararla.

Ejemplo clínico 1. «Si quieres que te abrace, has de ser buena niña y hacer las cosas bien». En este caso, se trata de una paciente con personalidad obsesiva y heridas melancólicas. Tras narrar la situación conflictiva con la madre, el mensaje implícito de esta última es de desvalorización, con pérdida de valor y de cariño si no hace las cosas bien.

Desde su rol de adulto, le pide a su madre lo que necesita, su apoyo, su cariño y su valor. La madre también funciona así en su vida, vemos que le gustaría valorarla, pero no puede. Hacemos reconstrucción yo adulto/yo niño. La invito a que, desde su rol de adulta, al dirigirse a la niña, le mire a ver si puede valorarla por quién es y no por las acciones que realiza. La paciente responde afirmativamente. También le invito a que le diga a su yo niña que no va a hacer falta que haga nada para tener el cariño de nadie.

Ejemplo clínico 2. «Cuando no me doy derecho a cuidar de mí»: las heridas melancólicas. Como he ido desarrollando, teniendo en cuenta las diversas patologías, nos hemos encontrado con diferentes maneras de proceder con esta técnica. A algunos pacientes, sobre todo melancólicos, paranoides y obsesivos, les cuesta entregarse a la situación de disfrute de la situación restauradora.

Citando y recordando algunas fantasías temidas de los pacientes y/o algunos miedos a repetir las vivencias sufridas anteriormente, los temores que crean estas resistencias a elaborar y restaurar están relacionados con no sentirse con derecho a entregarse al disfrute.

Por ejemplo, si las heridas son melancólicas y la dificultad es para darse derechos a sí mismos, en el espacio psicodramático aplicamos la técnica del espejo. Esta técnica consiste en colocar frente al paciente un objeto simbólico que le represente y pedirle que se observe desde fuera, como si fuese un observador externo. Después, le sugerimos que verbalice cuáles considera el paciente que son las dificultades que tiene para decirse cosas buenas, para cuidarse, ser buena madre consigo mismo o para darse derechos (Monzón, 2011).

Luego, llevamos a cabo la técnica de el yo adulto y el yo niño para que mire ahora, desde su rol adulto, si quiere darse esos derechos o, en cambio, le va a seguir rechazando y a continuar sin dárselos. Cuando el paciente, como adulto, vuelve a repetir las actitudes de incomprensión que criticaba de los otros, patrón denominado por Rosa Cuckier (1992) reveses de la defensa, el objetivo es guiarlo e intervenir indicándole, si es así, cómo se trataría a un niño de $\mathrm{x}$ años (para que empiece a activar sus partes de cuidado y no repetir el mismo patrón).

En último lugar, volvemos a la escena en la que recibió ese derecho en su vida y realizamos un cambio de rol con el yo niño, reforzando la vivencia de incorporación de esos mensajes.

\section{Conclusiones}

Las técnicas psicodramáticas del observador externo, la visualización y la concretización nos permiten la posibilidad de identificar y elaborar las heridas opresivas y carenciales creadas y agravadas a lo largo de la biografía de nuestros pacientes. El paciente puede visualizar qué tipo de dificultades y/o de miedos tiene para poder cuidarse a sí mismo.

Con estas técnicas psicodramáticas y respetando el proceso y las fases de la psicoterapia y la psicopatología psicodinámica, se pretende la reparación y elaboración de los conflictos, entendidos desde un lugar, en palabras de Bustos (1985) que debe ser desde lo sentido, y comprender desde la lógica lo vivido. 
La técnica psicodramática del yo grande y el yo pequeño nos facilita poder situarnos aproximadamente en el momento en que se crearon estas situaciones conflictivas o deficitarias. Es una técnica catártica y reparadora, pero lo más importante es que, además, crea y/o activa el sentimiento interno de capacidad, de cuidado de uno mismo y el sentimiento de logro. A través de esta técnica, los pacientes pueden crear la sensación de poder resolver la situación por sí mismos, de que hay solución y posibilidad de alivio del sufrimiento. Por último, es un paso que ayuda en el vínculo terapéutico a rematrizar las carencias básicas, el apoyo incondicional, de seguridad afectiva y de guía.

\section{Referencias}

Bustos, D. (1985). Nuevos rumbos en psicoterapia psicodramática. Individual, parejas y grupo en función social. La Plata, Argentina: Editorial Momento.

Cuckier, R. (1992). Psicodrama bipersonal. Sao Paulo, Brasil: Ágora.

Herranz, T. (2012). El final de un principio: el principio de un modo de pensar y hacer psicodrama, en Revista de Psicoterapia y Psicodrama, 1(1), 77-87.

Monzón, S. (2007). Psicoterapia psicodramática bipersonal. Reconstruyendo la identidad y los déficits afectivos en el TLP. Un nombre propio. Trabajo presentado en el VI Congreso Iberoamericano de Psicodrama «Psicodrama en síndromes y conflictos culturales», Palexco, La Coruña.

Monzón, S. (2011). El pensamiento psicoanalítico en la escena psicodramática. Trabajo presentado en las Jornadas de reflexión interna sobre psicoterapia dinámica y psicoanalítica. Asociación Española de Psicoanálisis Freudiano Oskar Pfister, Madrid.

Monzón, S. (2012). Introducción al trabajo de las resistencias desde la psicoterapia psicodramática bipersonal. Revista de Psicoterapia y Psicodrama, 1(1), 124-130.

Villamarzo, P. (1987). Curso general de técnica psicoanalítica, (Vol. I, II, III, IV y V), Salamanca, España: Ed. Instituto Superior de Estudios Freudianos Oskar Pfister.

Artículo recibido: 01/06/2018

Artículo aceptado: 15/10/2018 